ISAHP 1996, VANCOUVER, JULY 12-15, 1996

\title{
ON THE DECISION MAKER'S STRESS IN APPLYING ANALYTIC HIERARCHY PROCESS
}

\author{
Masatak̄e Nakanishi \\ Faculty of Economics \\ Nagoya Economics Universitiy, Uchikubo, Inuyama, Aichi, 484 Japan \\ mgg03324@niftyserve.or.jp \\ Eizo Kinoshita \\ Faculty of Urban Science \\ Meijo University, Nijigaoka, Kani, Gifu, 509-02 Japan \\ kinoshit@urban.meijo-u.ac.jp
}

\begin{abstract}
This study introduces a model for the decision maker's stress which occurs in the application of the Analytic Hierarchy Process (AHP). There are two types of decision maker's stress: (1) bugs in the course of comparisons, and (2) cognitive disposition or circular relation. The latter can be identified as a new independent value system. The decision maker's stress can be inspected by the Consistency Index and the mechanism analysed by means of phases of stress triangle transformations.
\end{abstract}

\section{Introduction}

The Analytic Hierarchy Process (AHP) is a well-known decision-making support tool developed by $T$. Saaty. It defines the problem being analysed as a hierarchical structure of criteria and elements.

\section{Requirements of Consistency Index (C.I.) in a Pairwise Comparison Method}

Saaty's pairwise comparison method aims to evaluate the value of contradicting elements by calculating the matrix in a manner that measures inconsistency. To do this, Saaty invented the Consistency Index (C.I.) to identify the suitability of pairwise comparisons. The C.I. is defined by the eigenvalue of a pairwise comparison matrix and the value equals zero when there is a perfect transitivity among the elements. C.I. becomes larger as inconsistency increases. For instance, when there is a complete transitivity as $\mathrm{A}>\mathrm{B}>\mathrm{C}>\mathrm{D}>\mathrm{E}, \mathrm{C} . \mathrm{I}$. $=0$. (Table 1 ).

Table 1 C.I. $=0$ Transitivity

$\begin{array}{lrrrrrr} & \text { A } & \text { B } & \text { C } & \text { D } & \text { E } & \text { Weights } \\ \text { A } & 1 & 2 & 4 & 8 & 16 & 0.5161 \\ \text { B } & & 1 & 2 & 4 & 8 & 0.2581 \\ \text { C } & & & 1 & 2 & 4 & 0.1290 \\ \text { D } & & & & 1 & 2 & 0.0645 \\ \text { E } & & & & & 1 & 0.0323\end{array}$


Saaty states by convention, that C.I. should be 0.1 or less. We have looked into a case to find out about the situation in which C.I. $>0.1$. Although there is a transitivity as $A>B>C>D>E$ in this case, due to some kind of cognitive disposition, C.I. $=0.1926$ (Table 2).

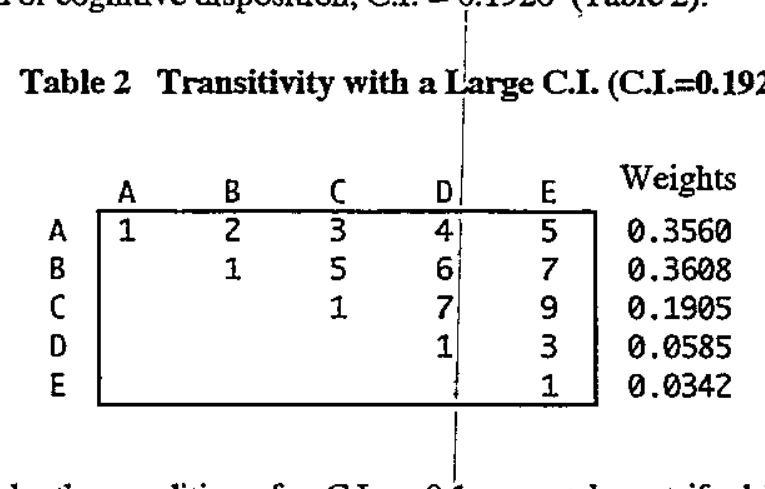

In other words, the conditions for C.I. $<0.1$ cannot be satsifted by merely maintaining the ordinal transitivity relation. And when we look into the case in which there is a defective transitivity, the C.I. indicates an unsatisfactory situation. A point in a pairwise comparison matrix (B vs. E) shows a defect in the transitivity relation among B, C, D, and E, and the C.I. is large: C.I. $=0.2549$ (Table 3).

Table 3 A Defective Transitivity (C.I. $=0.2549$ )

It seems as though Saaty duplicates the pairwise comparison matrix with a kind of score sheet always looking forward to a transitivity of C.I. $<0.1$ as the result of a set of comparisons. Does this requirement always match our actual cognitive mechänism?

\section{Circular Relation and Transitivity Relation}

One reason of the defective transitivity may be the cognitive disposition characteristic to all human-beings. We would not go into details of the human cognitive tendencies, however, all of you know that there are numerous psychological studies on this subject.

We would like to refer to the Circular Relation.

\section{(1) Circular Relation as a Game Rule}

The well known Japanese Janken Play of Stone, Scissors, Paper is an example of a circular relation. The triad match among Snake, Frog, and Slug is also of an oriental origin. The conflict is "settled" in a circular relation as Stone repels Scissors, Scissors cuts Paper, and Paper wraps Stone. Snake eats Frog, Frog eats Slug, but Slug repels Snake. As artist Taro Okamoto once said, "Let thesis and antithesis be consistent with each other. Let conflict stand by, because there is no problem with it." We may say that we Japanese are good at accepting a contradictory situation or a circular relation a priori. 
The criteria in a Janken Play are not identified on purpose. The hardness of the Stone, the sharpness of the Scissors, and the extensiveness of the Paper, respectively are criteria that are clearly competitive and unquestionable. Thus, there is no need of an examination or evaluation of criteria in a circular relation defined by a game rule.

\section{(2) Circuiar Relation in a Natural Attitude}

A circular relation is found not only in a situation related to rules and regulations, but also, in a situation related to the natural attitude of the decision maker.

When planning to eat out and given a choice between two cuisines, a person may prefer:

$$
\begin{aligned}
& \text { French > Japanese } \\
& \text { Chinese > French } \\
& \text { Japanese }>\text { Chinese }
\end{aligned}
$$

and may not be able to explain why. In this case, the criteria themselves cannot be identified.

\section{(3) Circular Relation Among Criteria}

If criteria for the evaluation can be clearly identified, there may be a transitivity according to each criteria in most cases. Such cases cannot be defined as examples of circular relation although they may seem to be circular at a first glance. These cases are subject to the application of the usual AHP, and the final solution depends on the distribution of the weights of the criteria. However, if the criteria themselves are in circular relation, they cannot be utilized for the solution (Table 4).

\section{Table 4 Circular Relation Generated from Evaluation of Cars}

$$
\begin{array}{ll}
\text { Cost }(\mathrm{C}) & : \text { Car-A }>\text { Car-B }>\text { Car-C } \\
\text { Status }(\mathrm{S}) & : \text { Car-B }>\text { Car-C }>\text { Car-A } \\
\text { Performance(P) } & : \text { Car-C }>\text { Car-A }>\text { Car-B }
\end{array}
$$

(Criteria Comparison)

\begin{tabular}{l|ccc|c}
\multicolumn{1}{c}{ Cost(C) } & $\mathrm{C}$ & $\mathrm{S}$ & $\mathrm{P}$ & Weights \\
\cline { 2 - 4 } Status(S) & 1 & 2 & $1 / 2$ & 0.3333 \\
Performance(P) & & 1 & 2 & 0.3333 \\
\cline { 2 - 3 } & & & 1 & 0.3333
\end{tabular}

\section{(4) Circular Relation by the Sum of Individual Evaluations}

There may be a transitivity within the individual, but when these are automatically summed, a circular relation may be generated.

Bill $\mathrm{A}>\mathrm{B}>\mathrm{C}$

Bob $\mathrm{B}>\mathrm{C}>\mathrm{A}$

Ryu $\mathrm{C}>\mathrm{A}>\mathrm{B}$ 
The above circular relation may be tacitly denied when we strictly follow Saaty's concept of the C.I. However, we would like to propose a consideration of the circular relation as a value system that is independent of and different from a transitivity relation.

C.I. has been usually applied to the quality assurance of pairwise comparisons. However, the value of the C.I. is increased not only by the defect of comparisons, but also by circular relations and cognitive dispositions. We should rather recognize C.I. as a clue for the inspection of such particular situations.

\section{Separation of Circular Relation}

\section{(1) Separation Procedure}

We propose a separation procedure in which the circular relation and the transitivity relation are separated as independent different systems from the original mixed relation (Figure 1).
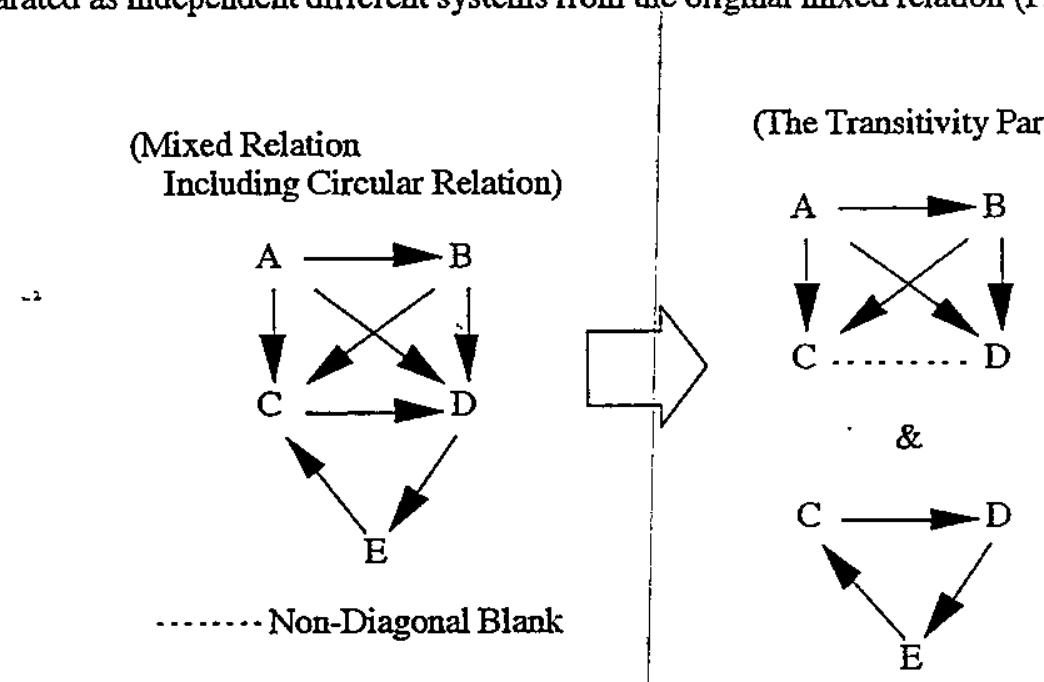

........ Non-Diagonal Blank

(The Circular Relation Part)

Figure 1 Image of Separation of Circular Relation

The separation procedure is as follows.

(a) C.I. Calculation.

(b) Identification of the Triad Circular Relation.

(c) Separation of the Triad Circular Relation (See Figure 1).

The Transitivity Part remains as an incomplete pairwise comparison matrix.

(d) Pairwise Comparisons in the Transitivity Part Only.

(e) Research of the Reason of the Triad Circular Relation. 
(2) Calculation of Incomplete Pairwise Comparison Matrix

We have adopted the Harker Approximation Method in calculating the incomplete pairwise comparison matrix, i.e., 0's are placed in the non-diagonal blanks, and the number of 0 's in each row is added to 1 in the corresponding diagonal position.

Table 5 Mixed Relation Matrix

\begin{tabular}{l|ccccc|c}
\multicolumn{1}{c}{ A } & B & C & D & E & Weights \\
\cline { 2 - 5 } & 1 & 2 & 4 & 8 & 16 & 0.5211 \\
B & & 1 & 2 & 4 & 8 & 0.2606 \\
C & & & 1 & 2 & $1 / 2$ & 0.0919 \\
D & & & & 1 & 2 & 0.0651 \\
E & & & & & 1 & 0.0613
\end{tabular}

According to the case shown in the previous figure, the Mixed Relation Matrix

(Table 5) will be separated into the Transitivity Part Matrix (Table 6) and the Triad Circular Relation Matrix (Table 8). The transitivity part includes non-diagonal blanks, and forms an incomplete pairwise comparison matrix which is transformed into a Harker Approximation Matrix (Table 7). X represents a non-diagonal blank in the following tables.

Table 6 Transitivity Part with blanks

Table 7 Harker Approximation Matrix

\begin{tabular}{|c|c|c|c|c|c|c|c|c|c|c|c|c|}
\hline & A & B & $c$ & D & $\mathrm{E}$ & & A & B & C & D & $E$ & Weights \\
\hline A & 1 & 2 & 4 & 8 & 16 & A & 1 & 2 & 4 & 8 & 16 & 0.5161 \\
\hline B & & 1 & 2 & 4 & 8 & B & & 1 & 2 & 4 & 8 & 0.2581 \\
\hline C & & & 1 & $x$ & $x$ & $C$ & & & 3 & 0 & 0 & 0.1290 \\
\hline D & & & $x$ & 1 & $x$ & D & & & 0 & 3 & 0 & 0.0645 \\
\hline$E$ & & & $\mathrm{x}$ & $x$ & 1 & $\mathrm{E}$ & & & 0 & 0 & 3 & 0.0323 \\
\hline
\end{tabular}

Table 8 Triad Circular Relation Part Separated From the Original Matrix

\begin{tabular}{lccc|c} 
& $C$ & $D$ & $E$ & Weights \\
\cline { 2 - 3 } C & 1 & 2 & $1 / 2$ & 0.3333 \\
& & 1 & 2 & 0.3333 \\
& & 1 & 0.3333
\end{tabular}

C. $I=0.2500$

\section{(3) Reduction to Circular Kernel}

The circular relation appears in various scales. However, any circular relation can be reduced to simple triangle either a circular triangle or a semi-circular triangle (including a 1:1 pair). We would like to name this circular triangle of elements as "circular kernel". Analysing large circles should start with the reduction to circular kemels (See Figure 2, 3). 
Arrows between the characters mean

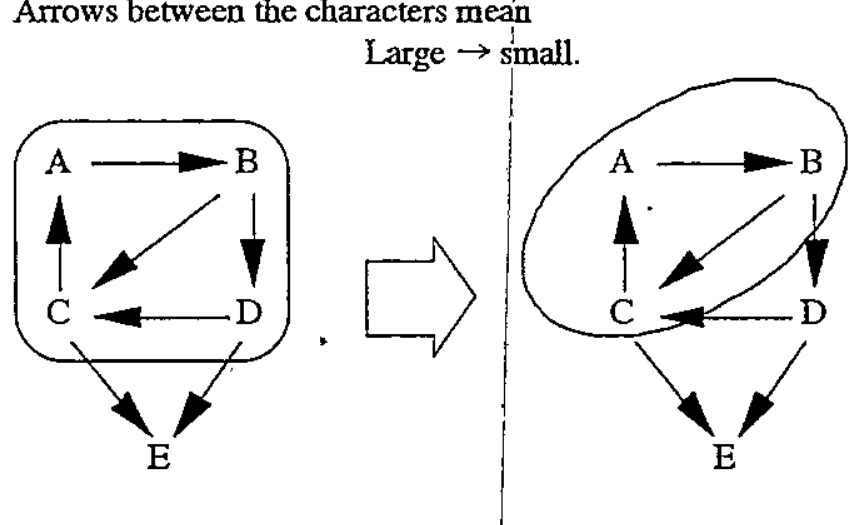

Figure 2 Reduction to a Circular Triangle of Elements

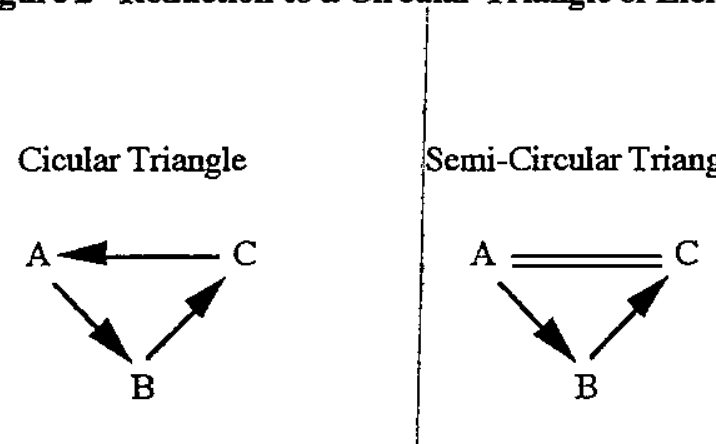

Figure 3 Two Types of Circular Kernels

\section{Consistency Index and the Decision Makèr's Stress}

\section{(1) Decision Maker's Stress}

The stronger the conflict, the larger the C.I. as shown below. Also, the C.I. is large when a strong conflict exists in the circular kernel of a mixed relation matrix (Tables 9, 10).

Table 9 C.I. is Large

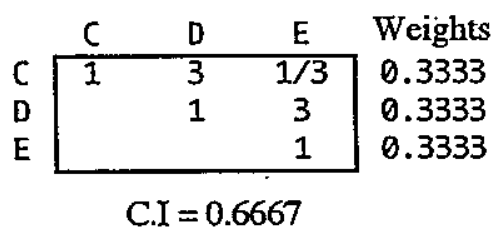

Table 10 C.I. is Very Large

\begin{tabular}{|c|c|c|c|c|}
\hline & 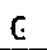 & D & $E$ & Weights \\
\hline C & 1 & 5 & $1 / 5$ & 0.3333 \\
\hline$D$ & & 1 & 5 & 0.3333 \\
\hline$E$ & & & 1 & 0.3333 \\
\hline
\end{tabular}

C.I $=1.6000$

We cannot define the degree of the decision maker's stress just by looking at the weight vectors, but the C.I. serves as an indicator of the size of the conflict. Or, as stated in section 2, the C.I. can be utilized as a scale to measure the degree of dilemma or a cognitive disharmony that occur in a decision making, i.e., the decision maker's stress. Let us further examine the mechanism of the decision maker's stress. 


\section{(2) Consistency Index of Equilateral Circular Triangle}

In order to conduct a fundamental analysis, we will start with an "equilateral circular triangle", a triad circulation with equal superiority. This triangle shows a $(3,3)$ pairwise comparison matirix (Table 11), and the consistency index is given by the formula: C.I. $=(\lambda \max -3) / 2$.

\section{Table 11 A Pairwise Comparison Matrix of the Equilateral Circular Triangle}

\begin{tabular}{|c|c|c|c|c|}
\hline & $x$ & $Y$ & $z$ & Weights \\
\hline$x$ & 1 & $\mathrm{P}$ & $1 / P$ & 0.333 \\
\hline$Y$ & & 1 & $\mathrm{P}$ & 0.333 \\
\hline & & & 1 & 0.33 \\
\hline
\end{tabular}
equation.

The eigenvalue $(\lambda \max )$ is given by the algebraic solution of the following characteristic

$$
\left|\begin{array}{ccc}
1-\lambda & \mathrm{P} & 1 / \mathrm{P} \\
1 / \mathrm{P} & 1-\lambda & \mathrm{P} \\
\mathrm{P} & 1 / \mathrm{P} & 1-\lambda
\end{array}\right|
$$

The maximum eigenvalue is given by the following formula.

$\lambda=1-\sqrt[3]{\frac{-\left(\mathrm{P}^{3}+1 / \mathrm{P}^{3}\right)+\sqrt{\left(\mathrm{P}^{3}+1 / \mathrm{P}^{3}\right)^{2}-4}}{2}}-\sqrt[3]{\frac{-\left(\mathrm{P}^{3}+1 / \mathrm{P}^{3}\right)-\sqrt{\left(\mathrm{P}^{3}+1 / \mathrm{P}^{3}\right)^{2}-4}}{2}}$

This eigenvalue indicates that as $P$ becomes larger, $\lambda$ approaches $1+P$, and almost equals it when $P$ is over 5. Actually, an equilateral circulation of $P=5$ may be the one with the strongest conflict we would ever experience (C.I. = 1.6).

\section{(3) Transition of Phases from Transitivity to Circulation via Disposition}

\section{(a) Transformation Phases of Stress Triangle}

We categorize the decision maker's stress into various groups shown as arrow graph triangles $\mathrm{ABC}$ of different shapes according to each phase of transition that corresponds with the $(3,3)$ pairwise comparison matrix (See Figure 4). The three sides ( $=$ "beam", $b=$ " "arm", and $c=$ "string") are defined as the entry of pairwise comparisons $(A \rightarrow B, B \rightarrow C, C \rightarrow A)$ on condition of $a \geq b$ and $b \geq c$. The lengths [a], $[\mathrm{b}]$, and $[\mathrm{c}]$ are given by the logarithm of $\mathrm{a}, \mathrm{b}$, and $\mathrm{c}$ as follows:

$$
\begin{aligned}
& {[\mathrm{a}]=\log \mathrm{a}} \\
& {[\mathrm{b}]=\log \mathrm{b}} \\
& {[\mathrm{c}]=\log \mathrm{c}}
\end{aligned}
$$


We define $\theta$ as the arm's external rotation angle from beam's production.

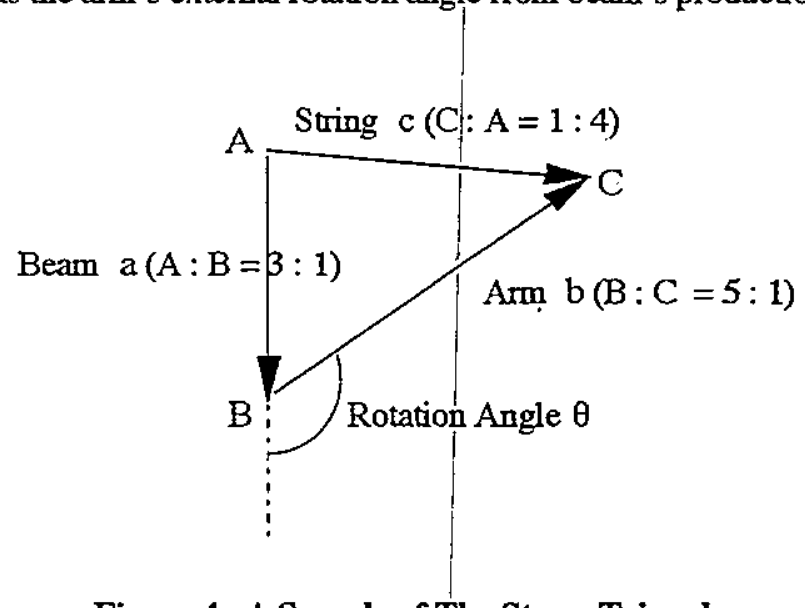

Figure 4 A Sample of The Stress Triangle

The stress triangle with the arm's rotation on condition of $[a]+[b] \geq[c]$ has 6 phases as follows: follows:

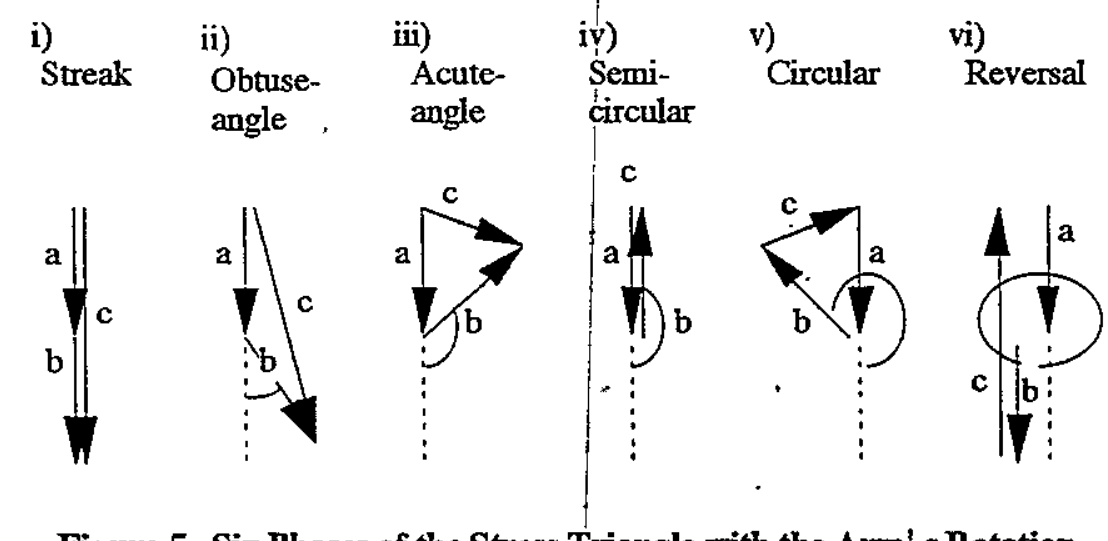

Figure 5 Six Phases of the Stress Triangle with the Arm's Rotation

These are grouped into tail-wind $(A \rightarrow C)$, dropped-wind $(A=C)$, head-wind $(A \leftarrow C)$ according to the arrow direction of side $\mathrm{c}$ on $\mathrm{A}$.

$<$ Tail-Wind

i) Streak $-\theta=0^{\circ}$

$--[$ beam $]+[\mathrm{arm}]=[$ string $]$. Stress is zero.

ii) Obtuse-angle triangle $\quad-0^{\circ}<\theta<90^{\circ}$

-- String is long enough. Stress is small.

iii) Acute-angle triangle $--90^{\circ}<\theta<180^{\circ}$

-. String is unsatisfactorily short. Stress is large.

$<$ Dropped-Wind $>$

iv) Semi-circular triangle -- $\theta=180^{\circ}$

-- String has no length. Stress is very large. 
$<$ Head-Wind $>$

v) Circular triangle $\quad--180^{\circ}<\theta<360^{\circ}$

-- Over-rotated. Stress is remarkablly large.

vi) Reversal $\quad-\theta=360^{\circ}$

- Full-rotated. Stress is the largest above all .

On condition of $[\mathrm{a}]+[\mathrm{b}]<[\mathrm{c}]$, the string of the stress triangle is bent into arc, which has 2 phases as follows:

(vii) Over Total

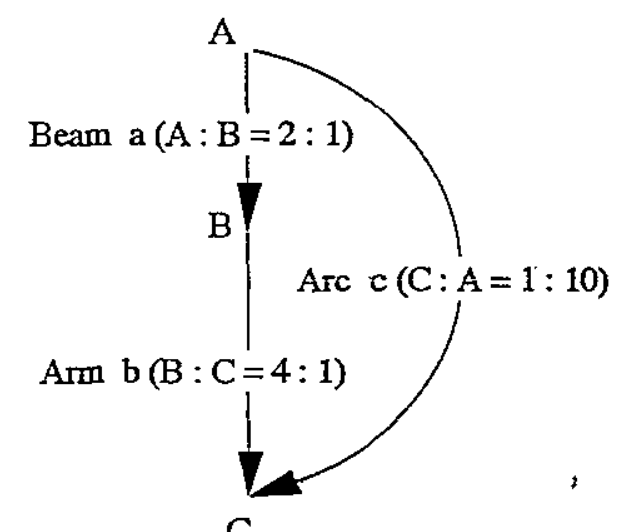

(viii) Over Reversal

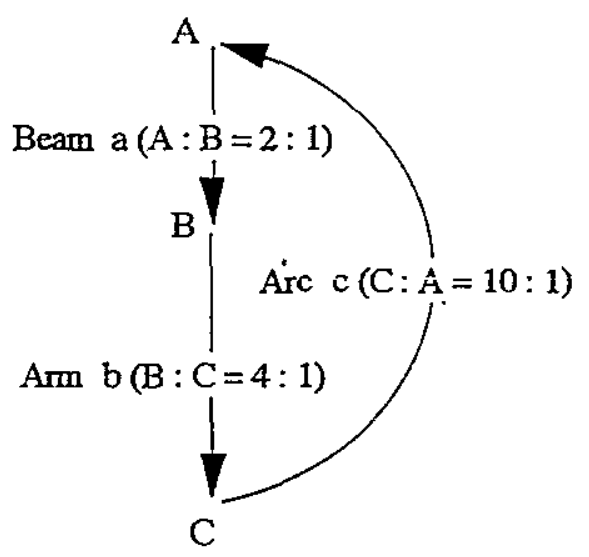

Figure 6 Two Phases of the Stress Triangle with an Arc

vii) Over total

-- the direction of string is the same as the beam and the arm.

viii) Over reversal

-- the direction of string is against the beam and the arm.

Therefore, the decision maker's stress has 8 phases with reference to the stress triangle transformation. (i) has a perfect consistency. (ii), (iii), and (vii) satisfy transitivity. (iv), (v), (vi), and (viii) require research of circular relation. The transitivity and the circular are usually regarded as two value systems which exclusively contradict each other. However, when we examine them utilizing the stress triangle transformation, we are able to see them as a result of a simple difference between cognitive phases.

\section{(b) C.I. of the Stress Triangle}

We will examine how the C.I. also changes in accordance with the transformation of the stress triangle. Let us look at a simple triangle of $[a]=2$ and $[b]=2$ on condition of $[a]+[b] \geq[c]$ for example (Table 12 and Figure 7). 
Table $12 \theta$, String, C.I. on condition of $a=b=2$

\begin{tabular}{|c|c|c|}
\hline rotation $\theta^{\circ}$ & string & C.I. \\
\hline 0 & 4.0000 & 0.0000 \\
\hline 30 & 3.8155 & 0.0001 \\
\hline 60 & 3.3220 & 0.0019 \\
\hline 90 & 2.6650 & 0.0092 \\
\hline 120 & 2.0000 & 0.0268 \\
\hline 150 & 1.4320 & 0.0592 \\
\hline 180 & 1.0000 & 0.1087 \\
\hline 210 & 0.6980 & 0.1742 \\
\hline 240 & 0.5000 & 0.2500 \\
\hline 270 & 0.3752 & 0.3276 \\
\hline 300 & 0.3010 & 0.3954 \\
\hline 330 & 0.2621 & 0.4448 \\
\hline 360 & 0.2500 & 0.4584 \\
\hline
\end{tabular}

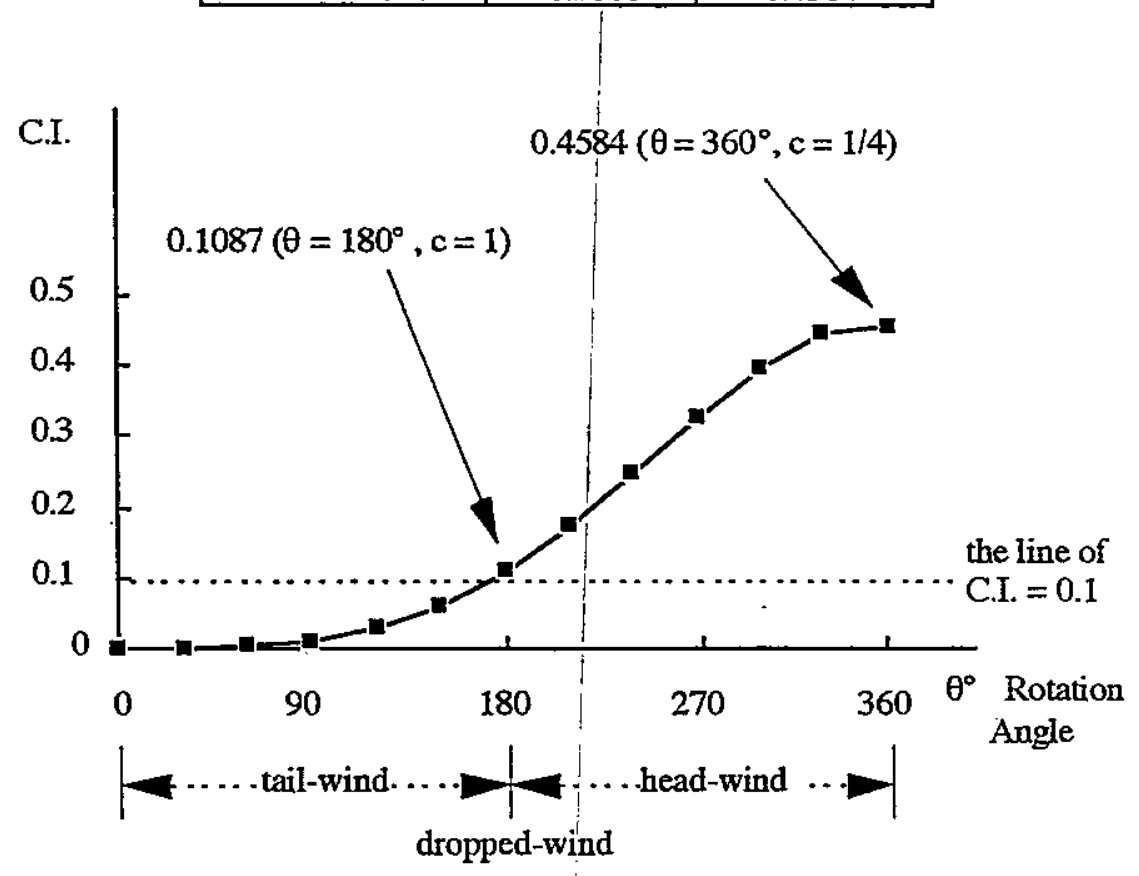

Figure 7 The Relation Between the Rotation Angle $\theta$ and C.I.

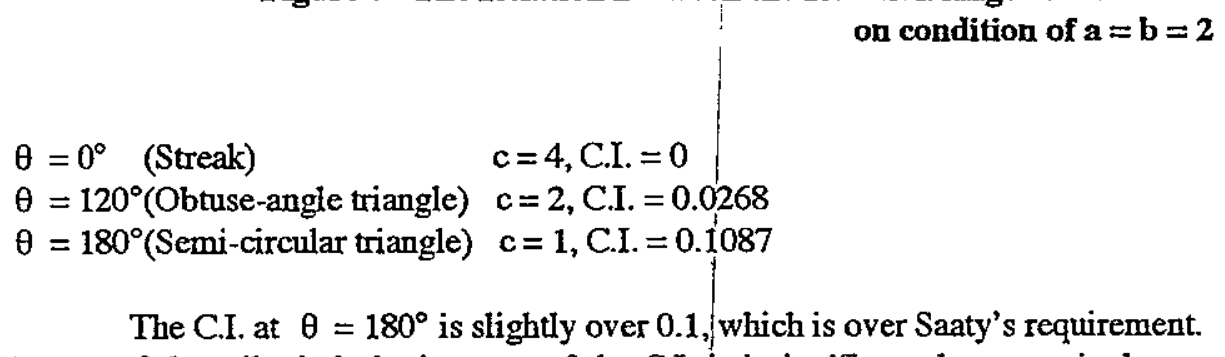

In case of the tail-wind, the increase of the C.I. is insignificant, however, it shows a large growth in case of the head-wind. 


$$
\begin{array}{ll}
\theta>180^{\circ} \text { (Circular triangle) } & c<1,0.1087<\text { C.I. }<0.4584 \\
\theta=360^{\circ} \text { (Reversal) } & c=1 /(A+B), \text { C.I. }=0.4584
\end{array}
$$

Let us focus our attention to the C.I. domain on $[\mathrm{a}]+[\mathrm{b}]<[\mathrm{c}]$. If we expand the arc, then C.I. grows larger toward the symptote value. In case of the over reversal, C.I. is given as $0.4584<$ C.I. $<$ $3.3919 \mathrm{max}$, and of the over total, $0<$ C.I. $<2.9107 \mathrm{max}$. However, C.I. of the over total triangle marks 0.1 just after $c>15$, which means that Saaty' $s$ criterion of inconsistency should be modified for this case.

The C.I. itself does not offer any explanation for the situation of the decision maker's stress, but we are able to analyse the situation utilizing the C.I. in connection with the stress triangle transformation.

\section{Errors and their Elimination from the Comparison Process}

Errors and bugs should be eliminated from the comparison process. Various types of quality assurance method, such as the cyclic feedback control, the random comparison entry, etc. are appllied for reliable comparisons. In those approaches, circular relation and cognitive disposition have been usually regarded as errors or entry bugs in the comparison process. However, when transitivity loses its absolute superiority, the evauluation of such out-of-transitivities may actually become a problem. If a decision maker has his or her own particular circular value system, or an intrinsic congitive disposition, then such systems should be reflected to the comparisons and not eliminated.

\section{Two Types of Decision Maker's Stress}

There are two types of decision maker's stress:

(1) Stress made by errors or bugs in the course of comparison.

(2) Stress made by circular relation or cognitive disposition.

(1) must be corrected. Many situations viewed as "circular relations" or "cognitive dispositions" are, in fact, defective systems made by errors or bugs, which can be corrected and improved. However, some situations truly comprise a circular relation and/or a cognitive disposition. When a stress is observed in a decision making procedure, it should be evaluated with a dual vision of (1) and (2) for its solution. The decision making stress can be an opportunity to discover a new value system.

\section{References}

1) Harker, P.T.(1987), "Incomplete pairwise comparisons in the analytic hierarchy process," Mathematical Modelling, Vol. 9, No. 11,837-848.

2) Saaty, T.L.(1980), The Analytic Hierarchy Process, Mcgraw-Hill. 